\title{
SMITH-TYPE STABILITY THEOREMS FOR THE DAMPED LINEAR OSCILLATOR
}

\author{
L. HATVANI \\ University of Szeged \\ Bolyai Institute \\ Aradi vértanúk tere 1, H-6720 Szeged, HUNGARY
}

\begin{abstract}
Sufficient conditions are given guaranteeing that every solution of the equation

$$
x^{\prime \prime}+h(t) x^{\prime}+\omega^{2} x=0 \quad(h(t) \geq 0, x \in \mathbb{R})
$$

and its derivative tend to zero as $t \rightarrow \infty$. The results are applicable in the general case $0 \leq 0 \leq h(t)<\infty$, i.e., conditions $h(t) \geq$ const. $>0$ and $h(t) \leq$ const. $<\infty$ are not required in general. In the first main theorem the damping is controlled on the whole half-line $[0, \infty)$. The second main theorem is devoted to the problem of the intermittent damping, when conditions are supposed only on the union of nonoverlapping intervals.
\end{abstract}

AMS Subject Classification: 34D20, 70J25

Key Words: asymptotic stability, growth condition, overdamping, intermittent damping, differential inequalities

Received: December 12, 2017; Accepted: March 5, 2018;

Published: March 12, $2018 \quad$ doi: $10.12732 /$ dsa.v27i2.6

Dynamic Publishers, Inc., Acad. Publishers, Ltd. https://acadsol.eu/dsa

\section{INTRODUCTION}

Consider the second order linear differential equation

$$
x^{\prime \prime}+h(t) x^{\prime}+\omega^{2} x=0 \quad(x \in \mathbb{R}),
$$

where the damping coefficient $h: \mathbb{R}_{+} \rightarrow \mathbb{R}_{+}$is a locally integrable function, $\mathbb{R}_{+}:=$ $[0, \infty)$, and the frequency $\omega>0$ is constant. We are interested in conditions of the asymptotic stability of the zero solution (of the equilibrium). In the case of (1) this 
means that every solution $x$ exists in $\mathbb{R}_{+}$and

$$
\lim _{t \rightarrow \infty} x(t)=\lim _{t \rightarrow \infty} x^{\prime}(t)=0 .
$$

R. A. Smith [16] proved the following important theorems.

Theorem A. Suppose that there exists a constant $\underline{h}>0$ such that

$$
h(t) \geq \underline{h}>0 \quad\left(t \in \mathbb{R}_{+}\right) .
$$

If

$$
\int_{0}^{\infty} e^{-H(t)} \int_{0}^{t} e^{H(s)} \mathrm{d} s \mathrm{~d} t \quad\left(H(t):=\int_{0}^{t} h\right),
$$

then the zero solution of (1) is asymptotically stable.

Theorem B. Suppose that there exists a sequence of intervals $\left\{I_{n}\right\}_{n=1}^{\infty}$ such that

$$
\sum_{n=1}^{\infty} m_{n}\left|I_{n}\right|\left(\min \left\{\left|I_{n}\right| ; \frac{1}{1+M_{n}}\right\}\right)^{2}=\infty
$$

where

$$
m_{n}:=\inf _{t \in I_{n}} h(t), M_{n}:=\sup _{t \in I_{n}} h(t), \quad\left|I_{n}\right| \text { denotes the length of } I_{n} .
$$

Then the zero solution of (1) is asymptotically stable.

It is easy to see that condition

$$
h(t) \leq \bar{h}<\infty \quad\left(t \in \mathbb{R}_{+}\right)
$$

implies (3). This means that Theorem A is a generalization of the classical theorem of J. J. Levin and J. A. Nohel [12] saying that conditions (2) and (5) together guarantee asymptotic stability. Since the appearance of this article a lot of papers have been published devoted to weakening conditions (2) and (5) (see, e.g., the papers $[1,3,4$, $5,6,7,8,9,10,13,14,15,16,17,18,19,20]$ and the references therein. If $(2)((5))$ is supposed, then we speak about large (small) damping; if neither (2) nor (5) are supposed, then the damping is called general. As is shown by experiences, to handle the general damping is essentially more difficult.

Smith [16] also proved that condition (3) is necessary for the asymptotic stability, so to improve Theorem A is possible only by weakening (2) not requiring the condition uniformly everywhere. For this reason there were introduced the notions of integral positivity and weak integral positivity (see $[3,19]$ ).

Definition 1. A locally integrable function $h: \mathbb{R}_{+} \rightarrow \mathbb{R}_{+}$is called 
a) integrally positive with parameter $\delta>0$ (in an abbreviated form $\operatorname{IP}(\delta)$ ) if

$$
\liminf _{t \rightarrow \infty} \int_{t}^{t+\delta} h>0
$$

b) weakly integrally positive with parameters $\delta>0$ and $\Delta \geq 0$ (in an abbreviated form $\operatorname{WIP}(\delta, \Delta))$ if for every sequence $\left\{t_{n}\right\}_{n=1}^{\infty}$

$$
t_{n}+\delta \leq t_{n+1} \leq t_{n}+\delta+\Delta \text { implies } \sum_{n=1}^{\infty} \int_{t_{n}}^{t_{n}+\delta} h=\infty .
$$

If (6) is satisfied for all $\delta>0((7)$ is satisfied for all $\delta>0$ and $\Delta \geq 0)$, then $h$ is called integrally positive (IP) (weakly integrally positive (WIP)).

Obviously, property IP is stronger than WIP; e.g., a decreasing function $h$ with $\lim _{t \rightarrow \infty} h(t)=0, \int_{0}^{\infty} h=\infty$ is WIP, but it is not IP.

In [9] we proved that (2) in Theorem A can be replaced with the condition that there is a $\delta \in(0, \pi / \omega)$ such that $h$ is $\operatorname{IP}(\delta)$, and the constant $\pi / \omega$ is sharp in this assertion. We also proved that if (5) is satisfied, i.e., for small damping, in Theorem A condition (2) can be replaced with the requirement that $h$ is $\operatorname{WIP}(\delta, \Delta)$ with some $\delta \in(0, \pi / \omega)$ and $\Delta \geq 0$, and the constant $\pi / \omega$ is sharp again. Recently [6] we constructed a counterexample showing that Theorem A does not remain true if we replace (2) with requiring weak integral positivity of $h$. In the first main theorem of the present paper we can replace condition (2) in Theorem A with one being somewhere between the integral positivity and weak integral positivity.

Theorem B is the first result about the problem of stability of the linear oscillators controlled by intermittent damping. This occurs if the system is positively damped in time intervals $I_{n}$, but the damping is either switched off or unrestricted at other times (we know only $h(t) \geq 0$ at these times). Our technique in the proof of the first main result is also suitable to deduce a sufficient condition generalizing theorem B, which will be our second main result. It will also work when $m_{n}=0$. To illustrate the result here we only mention that it makes possible to replace condition (4) with the following one: suppose that there exists a $\kappa \in(0,1)$ such that if $H_{n} \subset I_{n}$ is the union of finite intervals and $\operatorname{mes}\left(H_{n}\right) \geq \kappa\left|I_{n}\right|$, then we have

$$
\sum_{n=1}^{\infty}\left(\min \left\{\left|I_{n}\right| ; \frac{1}{1+M_{n}}\right\}\right)^{2} \int_{H_{n}} h=\infty .
$$

\section{LEMMAS}

By the new state variable $y=x^{\prime} / \omega$ equation (1) can be rewritten into the system

$$
x^{\prime}=\omega y, \quad y^{\prime}=-\omega x-h(t) y .
$$


On the phase plane $x, y$ we also use the polar coordinates $r, \varphi$ defined by

$$
x=r \cos \varphi, \quad y=r \sin \varphi \quad(r>0,-\infty<\varphi<\infty) .
$$

In these coordinates system (9) has the form

$$
\begin{aligned}
r^{\prime} & =-h(t) r \sin ^{2} \varphi, \\
\varphi^{\prime} & =-\omega-\frac{1}{2} h(t) \sin 2 \varphi .
\end{aligned}
$$

The solution with initial values $r\left(t_{0}\right)=r_{0}, \varphi\left(t_{0}\right)=\varphi_{0}$ will be denoted by $(r(t), \varphi(t))=$ $\left(r\left(t ; t_{0}, r_{0}, \varphi_{0}\right), \varphi\left(t ; t_{0}, \varphi_{0}\right)\right)$.

From the proof of Theorem A (see [16, 9]) it is clear that (3) is necessary and sufficient that $\lim _{t \rightarrow \infty} r(t)=0$ for all non-oscillatory solutions of (1), so we are searching for conditions guaranteeing the same property for every oscillatory solution.

The first lemma gives a lower and an upper estimate for the polar angle $\varphi(t)$ before and after a zero of $\sin \varphi(t)$, respectively. In the estimate there appears a transform function of $h$ defined by

$$
\tilde{h}\left(t ; t_{*}\right):=e^{-H(t)} \int_{t_{*}}^{t} e^{H(s)} \mathrm{d} s \quad\left(t_{*}, t \in \mathbb{R}_{+}\right),
$$

which plays an important role in what follows.

Lemma 2. If $T_{l}=T_{l}\left(t_{*}\right)>0, T_{r}=T_{r}\left(t_{*}\right)>0$ are so small that $-\pi \leq \varphi\left(t ; t_{*}, 0\right) \leq$ $\pi / 4$ on the interval $\left[t_{*}-T_{l}, t_{*}+T_{r}\right]$, then

$$
\begin{array}{ll}
\varphi\left(t ; t_{*}, 0\right) \geq-\omega \frac{\widetilde{2}}{\pi} h\left(t ; t_{*}\right) \geq 0 & \left(t_{*}-T_{l} \leq t \leq t_{*}\right), \\
\varphi\left(t ; t_{*}, 0\right) \leq-\omega \tilde{h}\left(t ; t_{*}\right) \leq 0 & \left(t_{*} \leq t \leq t_{*}+T_{r}\right) .
\end{array}
$$

Proof. For $\psi:=2 \varphi$ equation (11) yields the differential equation

$$
\psi^{\prime}=-2 \omega-h(t) \sin \psi
$$

Consider the regions

$$
\begin{aligned}
& D_{r}:=\left\{(t, \psi): t_{*} \leq t \leq t_{*}+T_{r},-2 \pi \leq \psi \leq 0\right\} \\
& D_{l}:=\left\{(t, \psi): t_{*}-T_{l} \leq t \leq t_{*}, 0 \leq \psi \leq \frac{\pi}{2}\right\} .
\end{aligned}
$$

Obviously, we have the estimates

$$
\begin{array}{ll}
-2 \omega-h(t) \sin \psi \leq-2 \omega-h(t) \psi & \left((t, \psi) \in D_{r}\right), \\
-2 \omega-h(t) \sin \psi \leq-2 \omega-h(t) \frac{2}{\pi} \psi & \left((t, \psi) \in D_{l}\right) .
\end{array}
$$


The unique solutions of the initial value problems

$$
\begin{array}{ll}
u^{\prime}=-2 \omega-h(t) u & \left(u\left(t_{*}\right)=0 ;(t, u) \in D_{r}\right), \\
u^{\prime}=-2 \omega-\frac{2}{\pi} h(t) u & \left(u\left(t_{*}\right)=0 ;(t, u) \in D_{l}\right)
\end{array}
$$

are

$$
\begin{array}{ll}
u_{r}(t):=-2 \omega \int_{t_{*}}^{t} e^{-(H(t)-H(s))} \mathrm{d} s & \left(t \geq t_{*}\right), \\
u_{l}(t):=-2 \omega \int_{t_{*}}^{t} e^{-\frac{2}{\pi}(H(t)-H(s))} \mathrm{d} s & \left(t \leq t_{*}\right),
\end{array}
$$

respectively. Applying the basic theorem of differential inequalities (for example, $[2$, Theorem 4.1 in Chapter III]) we get (13) and (14).

The second lemma provide us with a lower estimate for the distances of consecutive zeros of $\sin \varphi(t)$ for an oscillatory solution with $r(t) \not \rightarrow 0$ as $t \rightarrow \infty$.

Lemma 3. Let $\alpha, \varepsilon(0<\alpha<\pi / \omega, 0<\varepsilon<(\omega / 4)(\pi / \omega-\alpha))$ be given. If there exists a sequence $\left\{\left(u_{n}, v_{n}\right)\right\}_{n=1}^{\infty}$ such that

$$
\begin{gathered}
\varphi\left(v_{n}\right) \equiv-2 \varepsilon \quad(\bmod \pi), \varphi\left(u_{n}\right)-\varphi\left(v_{n}\right)=-\pi+4 \varepsilon \\
0<u_{n}-v_{n} \leq \alpha, \varphi\left(u_{n}\right) \leq \varphi(t) \leq \varphi\left(v_{n}\right)\left(v_{n} \leq t \leq u_{n}\right) \quad(n \in \mathbb{N}),
\end{gathered}
$$

then $\lim _{t \rightarrow \infty} r(t)=0$.

Proof. Integrating (11) we get the inequality

$$
\begin{aligned}
-\pi+4 \varepsilon & =\int_{v_{n}}^{u_{n}} \varphi^{\prime}(t) \mathrm{d} t=\int_{v_{n}}^{u_{n}}(-\omega-h(t) \sin \varphi(t) \cos \varphi(t)) \mathrm{d} t \\
& \geq-\omega\left(u_{n}-v_{n}\right)-\frac{\pi}{4 \varepsilon} \int_{v_{n}}^{u_{n}} h(t) \sin ^{2} \varphi(t) \mathrm{d} t .
\end{aligned}
$$

Therefore, the decrease of $r(t)$ admits the estimate

$$
\begin{aligned}
& \ln \frac{r\left(u_{n}\right)}{r\left(v_{n}\right)}=\int_{v_{n}}^{u_{n}} \frac{r^{\prime}(t)}{r(t)} \mathrm{d} t=-\int_{v_{n}}^{u_{n}} h(t) \sin ^{2} \varphi(t) \mathrm{d} t \\
& \quad \leq \frac{4 \varepsilon}{\pi}\left(-\pi+4 \varepsilon+\omega\left(u_{n}-v_{n}\right)\right) \leq \frac{4 \varepsilon \omega}{\pi}\left(-\left(\frac{\pi}{\omega}-\alpha\right)+\frac{4 \varepsilon}{\omega}\right)<0,
\end{aligned}
$$

whence $r(t) \rightarrow 0$ as $t \rightarrow \infty$.

Corollary 4. For an arbitrary solution let $\left\{\tau_{n}\right\}$ be the increasing sequence of all zeros of $\sin \varphi(t)$. If $\liminf _{n \rightarrow \infty}\left(\tau_{n+1}-\tau_{n}\right)<\pi / \omega$, then $\lim _{t \rightarrow \infty} r(t)=0$. 
In what follows, let $\left\{\tau_{n}\right\}_{n=1}^{\infty}$ denote the increasing sequence of all zeros of $\sin \varphi(t)$ corresponding to an oscillatory solution of (9). The third lemma says that $\varphi(t)$ remains far from $0(\bmod \pi)$ during $\left[t_{n}, s_{n}\right] \subset\left[\tau_{n}, \tau_{n+1}\right]$ uniformly with respect to $n$, provided that $\varphi\left(t_{n}\right) \equiv-2 \varepsilon, \varphi\left(s_{n}\right) \equiv-\pi+2 \varepsilon(\bmod \pi)$ with a fixed $\varepsilon>0$ and $r(t) \nrightarrow 0$ as $t \rightarrow \infty$.

Lemma 5. If for a fixed $\varepsilon(0<\varepsilon \leq \pi / 8)$ there exists a subsequence $\left\{n_{j}\right\}_{j=1}^{\infty}$ of the natural numbers and a sequence $\left\{\left(p_{j}, q_{j}\right)\right\}$ such that

$$
\begin{gathered}
\tau_{n_{j}} \leq q_{j}<p_{j} \leq \tau_{n_{j}+1}, \quad \varphi\left(q_{j}\right) \equiv-2 \varepsilon, \varphi\left(p_{j}\right) \equiv-\varepsilon \quad(\bmod \pi) \\
\varphi\left(q_{j}\right)<\varphi(t)<\varphi\left(p_{j}\right) \quad\left(q_{j}<t<p_{j}\right)
\end{gathered}
$$

for all $j$, then $\lim _{t \rightarrow \infty} r(t)=0$.

Proof. Integrating (11) we get

$$
\begin{aligned}
\varepsilon & =\int_{q_{j}}^{p_{j}} \varphi^{\prime}(t) \mathrm{d} t=\int_{q_{j}}^{p_{j}}(-\omega-h(t) \sin \varphi(t) \cos \varphi(t)) \mathrm{d} t \\
& \leq-\omega\left(p_{j}-q_{j}\right)+\cot \varepsilon \int_{q_{j}}^{p_{j}} h(t) \sin ^{2} \varphi(t) \mathrm{d} t .
\end{aligned}
$$

On the other hand, from (10) we have

$$
\begin{aligned}
\ln \frac{r\left(p_{j}\right)}{r\left(q_{j}\right)} & =\int_{q_{j}}^{p_{j}} \frac{r^{\prime}(t)}{r(t)} \mathrm{d} t=-\int_{q_{j}}^{p_{j}} h(t) \sin ^{2} \varphi(t) \mathrm{d} t \\
& \leq-\left(\varepsilon+\omega\left(p_{j}-q_{j}\right)\right) \frac{1}{\cot \varepsilon}<-\frac{\varepsilon}{\cot \varepsilon}<0,
\end{aligned}
$$

which means that $r(t) \rightarrow 0$ as $t \rightarrow \infty$.

\section{DAMPING ON THE WHOLE INTERVAL $[0, \infty)$}

Theorem 6. Suppose that the following conditions are satisfied:

(i)

$$
\int_{0}^{\infty} \tilde{h}(t ; 0) \mathrm{d} t=\int_{0}^{\infty} e^{-H(t)} \int_{0}^{t} e^{H(s)} \mathrm{d} s \mathrm{~d} t=\infty ;
$$

(ii) for a fixed $\alpha(\pi / 2 \omega<\alpha<\pi / \omega)$ and for an arbitrary sequence $\left\{\left(\tau_{n}, t_{n}, s_{n}\right)\right\}$ the properties

$$
\begin{aligned}
& t_{n} \geq \tau_{n}+\frac{1}{2}\left(\frac{\pi}{\omega}-\alpha\right), s_{n} \geq t_{n}+\alpha ; \\
& 0<\tau_{n+1}-s_{n} \leq \frac{1}{2}\left(\frac{\pi}{\omega}-\alpha\right) \quad(n \in \mathbb{N})
\end{aligned}
$$

imply that at least one of the following three divergences holds: 
(a) $\sum_{n=1}^{\infty} \int_{\tau_{n}}^{t_{n}} h(t) \tilde{h}\left(t ; \tau_{n}\right)^{2} \mathrm{~d} t=\infty$;

(b) $\sum_{n=1}^{\infty} \int_{t_{n}}^{s_{n}} h(t) \mathrm{d} t=\infty$;

(c) $\sum_{n=1}^{\infty} \int_{s_{n}}^{\tau_{n+1}} h(t)\left(\tau_{n+1}-t\right)^{2} \mathrm{~d} t=\infty$.

Then the zero solution of (1) is asymptotically stable.

Proof. Smith [16] proved that (1) has non-oscillatory solutions not tending to zero as $t \rightarrow \infty$ if and only if $\int_{0}^{\infty} \tilde{h}(t ; 0) \mathrm{d} t<\infty$ (a simple proof can be found in [9]). So we have to prove only that every oscillatory solution tends to zero. Suppose the contrary, fix an oscillatory solution with $r_{\infty}:=\lim _{t \rightarrow \infty} r(t)>0$, and denote by $\left\{\tau_{n}\right\}_{n=1}^{\infty}$ the increasing sequence of all zeros of $\sin \varphi(t)$ for this solution. Starting from (10) we will come to a contradiction proving that

$$
\begin{aligned}
r\left(t_{0}\right)-r_{\infty} & =\int_{t_{0}}^{\infty} r(t) h(t) \sin ^{2} \varphi(t) \mathrm{d} t \geq r_{\infty} \int_{t_{0}}^{\infty} h(t) \sin ^{2} \varphi(t) \mathrm{d} t \\
& =r_{\infty} \sum_{n=1}^{\infty} \int_{\tau_{n}}^{\tau_{n+1}} h(t) \sin ^{2} \varphi(t) \mathrm{d} t=\infty .
\end{aligned}
$$

Let us fix $\varepsilon:=\pi / 4-\alpha \omega / 4 \leq \pi / 4-(\pi / 2 \omega) \omega / 4=\pi / 8$, and define $t_{n}, s_{n}$ so that

$$
\begin{aligned}
& t_{n}:=\min \left\{t \in\left(\tau_{n}, \tau_{n+1}\right): \varphi(t)-\varphi\left(\tau_{n}\right)=-2 \varepsilon\right\}, \\
& s_{n} \in\left(\tau_{n}, \tau_{n+1}\right), \varphi\left(s_{n}\right)-\varphi\left(\tau_{n+1}\right)=2 \varepsilon .
\end{aligned}
$$

By (11), fore every $n \in \mathbb{N}$ such $t_{n}, s_{n}$ exist, they are unique and possess the following properties:

$$
t_{n}-\tau_{n} \geq \frac{2 \varepsilon}{\omega}=\frac{1}{2}\left(\frac{\pi}{\omega}-\alpha\right), \tau_{n+1}-s_{n} \leq \frac{2 \varepsilon}{\omega}=\frac{1}{2}\left(\frac{\pi}{\omega}-\alpha\right) \quad(n \in \mathbb{N}) .
$$

On the other hand, by Lemma 3 we know that $s_{n}-t_{n} \geq \alpha$ for all $n$ large enough. Therefore, without loss of the generality, we can say that the sequence $\left\{\left(\tau_{n}, t_{n}, s_{n}\right)\right\}$ possess all the properties (17).

Using the notations introduced in (19) we disintegrate the $n$ 'th member of the sum in (18) into three parts:

$$
\int_{\tau_{n}}^{\tau_{n+1}} h(t) \sin ^{2} \varphi(t) \mathrm{d} t=\int_{\tau_{n}}^{t_{n}} \ldots+\int_{t_{n}}^{s_{n}} \ldots+\int_{s_{n}}^{\tau_{n+1}} \ldots=: I_{1}^{n}+I_{2}^{n}+I_{3}^{n} .
$$

Now we estimate these three integrals from below. Applying Lemma 2 we obtain

$$
\begin{aligned}
I_{1}^{n} & =\int_{\tau_{n}}^{t_{n}} h(t) \sin ^{2} \varphi(t) \mathrm{d} t \geq \frac{4}{\pi^{2}} \int_{\tau_{n}}^{t_{n}} h(t) \varphi^{2}(t) \mathrm{d} t \\
& \geq \frac{4 \omega^{2}}{\pi^{2}} \int_{\tau_{n}}^{t_{n}} h(t) \tilde{h}\left(t ; \tau_{n}\right)^{2} \mathrm{~d} t .
\end{aligned}
$$


For the second integral, Lemma 5 yields the inequalities

$$
\varphi\left(\tau_{n}\right)-\varepsilon>\varphi(t)>\varphi\left(\tau_{n+1}\right)+\varepsilon \quad\left(t_{n} \leq t \leq s_{n}\right)
$$

from which we obtain

$$
\begin{aligned}
I_{2}^{n} & =\int_{t_{n}}^{s_{n}} h(t) \sin ^{2} \varphi(t) \mathrm{d} t \geq \sin ^{2} \varepsilon \int_{t_{n}}^{s_{n}} h(t) \mathrm{d} t \\
& \geq \frac{\omega^{2}}{4 \pi^{2}}\left(\frac{\pi}{\omega}-\alpha\right)^{2} \int_{t_{n}}^{s_{n}} h .
\end{aligned}
$$

Finally, from Lemma 2 we get

$$
\begin{aligned}
\sin ^{2} \varphi(t) & \geq\left(\frac{2}{\pi}\right)^{2} \varphi^{2}(t) \geq \frac{4 \omega^{2}}{\pi^{2}}\left(\frac{\widetilde{2}}{\pi} h\left(t ; \tau_{n+1}\right)\right)^{2} \\
& =\frac{4 \omega^{2}}{\pi^{2}}\left(\int_{t}^{\tau_{n+1}} e^{\frac{2}{\pi}(H(s)-H(t))} \mathrm{d} s\right)^{2} \geq \frac{4 \omega^{2}}{\pi^{2}}\left(\tau_{n+1}-t\right)^{2}
\end{aligned}
$$

and the third estimate

$$
I_{3}^{n} \geq \frac{4 \omega^{2}}{\pi^{2}} \int_{s_{n}}^{\tau_{n+1}} h(t)\left(\tau_{n+1}-t\right)^{2} \mathrm{~d} t .
$$

Summing up we have

$$
\begin{aligned}
& \int_{\tau_{n}}^{\tau_{n+1}} h(t) \sin ^{2} \varphi(t) \mathrm{d} t \geq c_{1}\left(\int_{\tau_{n}}^{t_{n}} h(t) \tilde{h}\left(t ; \tau_{n}\right)^{2} \mathrm{~d} t\right. \\
& \left.\quad+\int_{t_{n}}^{s_{n}} h(t) \mathrm{d} t+\int_{s_{n}}^{\tau_{n+1}} h(t)\left(\tau_{n+1}-t\right)^{2} \mathrm{~d} t\right) ; \\
& c_{1}:=\frac{\omega^{2}}{4 \pi^{2}} \min \left\{1 ;\left(\frac{\pi}{\omega}-\alpha\right)\right\} .
\end{aligned}
$$

The second condition of the theorem implies (18), that is a contradiction.

Earlier theorems concentrated only on divergence (b). The following question arises in connection with condition (ii) in Theorem 6: is it possible that (a) or (c) are satisfied but (b) is not? The answer is affirmative.

Example 7. We consider the damping coefficient

$$
h(t):=\left\{\begin{array}{ll}
1 & \text { if } 3 i \pi-\pi \leq t<3 i \pi+\pi \\
0 & \text { if } 3 i \pi+\pi \leq t<3 i \pi+2 \pi
\end{array} \quad(i \in \mathbb{N})\right.
$$

and equation (1) with $h$ defined in (21) and with $\omega=1$. We show that for an appropriate sequence $\left\{\left(\tau_{n}, t_{n}, s_{n}\right)\right\}$ and for all $\alpha$ divergence (a) is satisfied but (b) is not ; moreover, for every $\alpha$ and for every sequence $\left\{\left(\tau_{n}, t_{n}, s_{n}\right)\right\}$ at least one of 
divergences (a), (b), and (c) is satisfied, i.e., the zero solution of (1) with (21) is asymptotically stable.

In fact, if $\tau_{n}=3 n \pi, t_{n}=3 n \pi+\pi, s_{n}=3 n \pi+2 \pi$, then for arbitrary $\alpha$ conditions (17) are satisfied, divergence (a) is also satisfied, but (b) is not. On the other hand, let $\alpha \in(\pi / 2, \pi)$ arbitrarily fixed and $\left\{\tau_{n}, t_{n}, s_{n}\right\}$ be arbitrary. We show that at least one of the divergences (a) and (b) is satisfied. If $t_{n} \in[3 i \pi, 3 i \pi+\pi+(\pi-\alpha) / 4]$ for some $i \in \mathbb{N}$, then

$$
\begin{aligned}
\int_{\tau_{n}}^{t_{n}} h(t)\left(\tilde{h}\left(t ; \tau_{n}\right)\right)^{2} \mathrm{~d} t & \geq \int_{t_{n}-(\pi-\alpha) / 2}^{t_{n}} h(t)\left(\tilde{h}\left(t ; \tau_{n}\right)\right)^{2} \mathrm{~d} t \\
& \geq \int_{0}^{(\pi-\alpha) / 4}\left(1-e^{-u}\right)^{2} \mathrm{~d} u=: c_{2}>0 .
\end{aligned}
$$

If $t_{n} \geq(3 i+1) \pi+(\pi-\alpha) / 4$, then $s_{n} \geq(3 i+1) \pi$ $+(\pi-\alpha) / 4+\alpha=(3 i+2) \pi-3(\pi-\alpha) / 4$, therefore

$$
\begin{gathered}
s_{n+1} \geq(3 i+2) \pi-\frac{3}{4}(\pi-\alpha)+\left(\frac{1}{2}(\pi-\alpha)+\alpha\right)=(3 i+3) \pi \\
-\frac{5}{4}(\pi-\alpha) \geq(3 i+3) \pi-\frac{5}{4}\left(\pi-\frac{\pi}{2}\right)=(3 i+2) \pi+\frac{3}{8} \pi,
\end{gathered}
$$

hence

$$
\int_{t_{n+1}}^{s_{n+1}} h(t) \mathrm{d} t \geq \min \left\{\frac{3}{8} \pi ; \alpha\right\}=\frac{3}{8} \pi=: c_{3} .
$$

This means that

$$
\sum_{m=1}^{\infty} \max \left\{\int_{\tau_{2 m}}^{t_{2 m}} h(t)\left(\tilde{h}\left(t ; \tau_{2 m}\right)^{2} \mathrm{~d} t ; \int_{t_{2 m+1}}^{s_{2 m+1}} h(t) \mathrm{d} t\right\}=\infty\right.
$$

which implies that either

$$
\sum_{m=1}^{\infty} \int_{\tau_{2 m}}^{t_{2 m}} h(t)\left(\tilde{h}\left(t ; \tau_{2 m}\right)^{2} \mathrm{~d} t=\infty\right.
$$

or

$$
\sum_{m=1}^{\infty} \int_{t_{2 m+1}}^{s_{2 m+1}} h(t) \mathrm{d} t=\infty
$$

so one of divergences (a) and (b) is satisfied.

It can be verified that the same assertions are true for equation (1) with the unbounded damping coefficient

$$
h(t):=\left\{\begin{array}{ll}
i & \text { if } 3 i \pi-\pi \leq t<3 i \pi+\pi \\
0 & \text { if } 3 i \pi+\pi \leq t<3 i \pi+2 \pi
\end{array} \quad(i \in \mathbb{N}) ;\right.
$$

only constants $c_{2}$ and $c_{3}$ have to be modified in (22) and (23), respectively. Besides, one has to take into account that (16) is satisfied if the function $(t, s) \mapsto \exp [-(H(t)-$ 
$H(s))$ ] of two variables is identically zero on triangles $\left\{(t, s) \in \mathbb{R}^{2}:(3 i+1) \pi \leq t<\right.$ $(3 i+2) \pi,(3 i+1) \pi<s<t\}(i \in \mathbb{N})$.

It is worth noticing that earlier theorems controlling the damping on the whole interval $[0, \infty)$ cannot be applied to guarantee asymptotic stability for equation (1) with $(21)$ or $(24)$.

In the first corollary we replace divergence (a) with a more explicit one.

Corollary 8. Suppose that the following conditions are satisfied:

(i) (16) holds.

$\left(i i^{\prime}\right)$ For a fixed $\alpha(\pi / 2 \omega<\alpha<\pi / \omega)$ and for an arbitrary sequence $\left\{\left(\tau_{n}, t_{n}, s_{n}\right)\right\}$ introduce the notation $K_{n}:=\sup _{\left[\tau_{n}, \tau_{n+1}\right]} h$. Assume that the properties

$$
\begin{aligned}
& \frac{1}{2}\left(\frac{\pi}{\omega}-\alpha\right) \leq t_{n}-\tau_{n} \leq c_{4} K_{n}+c_{5} ; \quad s_{n}-t_{n} \geq \alpha ; \\
& \frac{c_{6}}{c_{7}+K_{n}} \leq \tau_{n+1}-s_{n} \leq \frac{1}{2}\left(\frac{\pi}{\omega}-\alpha\right) \quad(n \in \mathbb{N})
\end{aligned}
$$

with some positive constants $c_{4}, \ldots, c_{7}$ imply that at least one of the following three divergences holds:

(a) $\sum_{n=1}^{\infty}\left(\frac{1}{K_{n}^{2}} \int_{\tau_{n}}^{t_{n}} h(t)\left(1-e^{-K_{n}\left(t-\tau_{n}\right)}\right)^{2} \mathrm{~d} t\right)=\infty$;

(b) $\sum_{n=1}^{\infty} \int_{t_{n}}^{s_{n}} h(t) \mathrm{d} t=\infty$;

(c) $\sum_{n=1}^{\infty} \int_{s_{n}}^{\tau_{n+1}} h(t)\left(\tau_{n+1}-t\right)^{2} \mathrm{~d} t=\infty$.

Then the zero solution of (1) is asymptotically stable.

Proof. By the definitions of $\tilde{h}$ and $K_{n}$ we have the inequality

$$
\begin{aligned}
\tilde{h}\left(t ; \tau_{n}\right) & =\int_{\tau_{n}}^{t} e^{-(H(t)-H(s))} \mathrm{d} s \geq \int_{\tau_{n}}^{t} e^{-K_{n}(t-s)} \mathrm{d} s \\
& =\frac{1}{K_{n}}\left(1-e^{-K_{n}\left(t-\tau_{n}\right)}\right) \quad\left(t \in\left[\tau_{n}, t_{n}\right]\right),
\end{aligned}
$$

so the divergence (a) in Corollary 8 implies that in Theorem 6.

Considering again $t_{n}, s_{n}$ defined by (19), integrating (11) and applying the Schwarz Inequality we obtain

$$
\omega\left(t_{n}-\tau_{n}\right)-2 \varepsilon \leq\left(\int_{\tau_{n}}^{t_{n}} h\right)^{\frac{1}{2}}\left(\int_{\tau_{n}}^{t_{n}} h(t) \sin ^{2} \varphi(t) \mathrm{d} t\right)^{\frac{1}{2}} .
$$

Taking into account equation (11) and the definition of $K_{n}$ we get the inequality

$$
\omega\left(t_{n}-\tau_{n}\right)-\left(\ln \frac{r\left(\tau_{1}\right)}{r_{\infty}}\right)^{\frac{1}{2}} K_{n}^{\frac{1}{2}}\left(t_{n}-\tau_{n}\right)^{\frac{1}{2}}-2 \varepsilon \leq 0,
$$


from which there follows the existence of constants $c_{4}, c_{5}$ independent of $n$ (depending only on the solution) such that

$$
t_{n}-\tau_{n} \leq c_{4} K_{n}+c_{5} \quad(n \in \mathbb{N})
$$

On the other hand, integration of (11) yields

$$
-2 \varepsilon=\int_{s_{n}}^{\tau_{n+1}} \varphi^{\prime} \geq-\omega-K_{n}\left(\tau_{n+1}-s_{n}\right),
$$

whence

$$
\frac{c_{6}}{c_{7}+K_{n}} \leq \tau_{n+1}-s_{n} \quad(n \in \mathbb{N})
$$

with constants $c_{6}, c_{7}$ independent of $n$.

Several earlier results in the literature follow from Theorem 6 and Corollary 8. For example, if $h$ has the property $I P(\alpha)$ for some $\alpha \in(0, \pi / \omega)$ (especially, if (2) is satisfied), then divergence (b) holds, so Smith's Theorem A and Theorem 3.1 in [9] are corollaries of Theorem 6 . To deduce further corollaries we need an elementary lemma.

Lemma 9. If $\kappa>1, M>0$, then

$$
\frac{1}{M}\left(1-e^{-M(t-\tau)}\right) \geq \frac{1}{\kappa}(t-\tau) \quad\left(\tau<t<\tau+\frac{\ln \kappa}{M}\right) .
$$

Proof. The function on the left-hand side is concave and its derivative at $t=\tau$ equals 1 . The estimate is true to the right from $\tau$ as far as that the derivative of the function becomes smaller than $1 / \kappa$.

The following corollary is about the case of small damping (see [9, Theorem 4.1]).

Corollary 10. Suppose that

$\left(i^{\prime}\right) h$ is bounded on $[0, \infty)$;

$\left(i i^{\prime \prime}\right)$ for every sequence $\left\{\tau_{n}\right\}_{n=1}^{\infty}$ of the properties $\liminf _{n \rightarrow \infty}\left(\tau_{n+1}-\tau_{n}\right) \geq \pi / \omega, \tau_{n+1}-\tau_{n} \leq 2 \pi / \omega(n \in \mathbb{N})$ the divergence

$$
\sum_{n=1}^{\infty} \int_{\tau_{n}}^{\tau_{n+1}} h(t)\left(\min \left\{t-\tau_{n} ; \tau_{n+1}-t\right\}\right)^{2} \mathrm{~d} t=\infty
$$

holds,

then the zero solution of (1) is asymptotically stable. 
Proof. It is easy to see that if $h$ is bounded, then (16) holds. Suppose (5) and let us given a sequence $\left\{\left(\tau_{n}, t_{n}, s_{n}\right)\right\}$ satisfying $(25)$. Then there exists a constant $\Delta$ such that $t_{n}-\tau_{n} \leq \Delta(n \in \mathbb{N})$. Using Lemma 9 with $\kappa:=e^{\bar{h} \Delta}$, we can estimate further the left-hand side of divergence (a) in Corollary 8:

$$
\begin{aligned}
& \sum_{n=1}^{\infty}\left(\frac{1}{K_{n}^{2}} \int_{\tau_{n}}^{t_{n}} h(t)\left(1-e^{-K_{n}\left(t-\tau_{n}\right)}\right)^{2} \mathrm{~d} t\right) \\
& \quad \geq \frac{1}{\kappa^{2}} \sum_{n=1}^{\infty} \int_{\tau_{n}}^{t_{n}} h(t)\left(t-\tau_{n}\right)^{2} \mathrm{~d} t,
\end{aligned}
$$

provided that $t_{n}-\tau_{n} \leq \ln \kappa / K_{n}$ for all $n$. But $\ln \kappa / K_{n}=\bar{h} \Delta / K_{n} \geq \Delta$, and $t_{n}-\tau_{n} \leq$ $\Delta$ for all $n$, therefore (27) is true. Consequently, at least one of divergences (a), (b), (c) in Corollary 8 holds if

$$
\sum_{n=1}^{\infty} \int_{\tau_{n}}^{\tau_{n+1}} h(t)\left(\min \left\{1 ; t-\tau_{n} ; \tau_{n+1}-t\right\}\right)^{2} \mathrm{~d} t=\infty .
$$

We show that (ii") implies (28) for every sequence $\left\{\tau_{n}\right\}$ of the property $\lim \inf _{n \rightarrow \infty}\left(\tau_{n+1}-\right.$ $\left.\tau_{n}\right) \geq \pi / \omega$. If $\tau_{n+1}-\tau_{n}>2 \pi / \omega$ for some $n$, then we add the points $\tau_{n j}:=\tau_{n}+j \pi / \omega$ $(j=1,2, \ldots)$ until $\tau_{n+1}-\tau_{n j}$ becomes smaller than $2 \pi / \omega$. If $\left\{\tau_{m}^{\prime}\right\}_{m=1}^{\infty}$ denote the modified sequence, then

$$
\begin{aligned}
& \sum_{n=1}^{\infty} \int_{\tau_{n}}^{\tau_{n+1}} h(t)\left(\min \left\{1 ; t-\tau_{n} ; \tau_{n+1}-t\right\}\right)^{2} \mathrm{~d} t \\
& \quad \geq \sum_{m=1}^{\infty} \int_{\tau_{m}^{\prime}}^{\tau_{m+1}^{\prime}} h(t)\left(\min \left\{1 ; t-\tau_{m}^{\prime} ; \tau_{m+1}^{\prime}-t\right\}\right)^{2} \mathrm{~d} t \\
& \quad \geq \min \left\{1 ; \frac{\omega^{2}}{\pi^{2}}\right\} \sum_{m=1}^{\infty} \int_{\tau_{m}^{\prime}}^{\tau_{m+1}^{\prime}} h(t)\left(\min \left\{t-\tau_{m}^{\prime} ; \tau_{m+1}^{\prime}-t\right\}\right)^{2} \mathrm{~d} t \\
& \quad=\infty
\end{aligned}
$$

because of $\left(\mathrm{i}^{\prime \prime}\right)$.

\section{INTERMITTENT DAMPING}

The importance of Theorem B can be illustrated by Example 7: condition (4) is obviously satisfied for both damping coefficients (21) and (24), so Theorem B guarantees asymptotic stability in both cases. At the same time, earlier theorems in the literature cannot be applied, even the application of Theorem 6 is not trivial. (Actually, if we modify definitions (21) and (24) requiring that $h(t)=0$ for rational $t$ 's, then Theorem B cannot be applied either, but Theorem 6 can.) This example shows that it is also important to generalize and to further develop the idea appearing in Theorem B. 
Theorem 11. Suppose that there exists a sequence of non-overlapping intervals $\left\{I_{n}\right\}_{n=1}^{\infty}$ such that $\left|I_{n}\right| \leq \pi / 2 \omega(n \in \mathbb{N})$, and for every sequence $\left\{\xi_{n} \in \overline{I_{n}}\right\}$

$$
\sum_{n=1}^{\infty} \int_{I_{n}} h(t)\left(\min \left\{\left|t-\xi_{n}\right| ; \frac{1}{1+M_{n}}\right\}\right)^{2} \mathrm{~d} t=\infty
$$

is satisfied, where $M_{n}:=\sup _{t \in I_{n}} h(t)$, and $\overline{I_{n}}$ denotes the closure of $I_{n}$.

Then the zero solution of (1) is asymptotically stable.

Proof. Suppose that the assertion of the theorem is not true, i.e., there exists a solution $x$ such that $r_{\infty}>0$. We will come to a contradiction similarly to (18) proving that

$$
\begin{aligned}
r\left(t_{0}\right)-r_{\infty} & =\int_{t_{0}}^{\infty} r(t) h(t) \sin ^{2} \varphi(t) \mathrm{d} t \geq r_{\infty} \sum_{n=1}^{\infty} \int_{I_{n}} h(t) \sin ^{2} \varphi(t) \mathrm{d} t \\
& =\infty
\end{aligned}
$$

There are two cases:

Case A: $x$ is oscillatory. Let $\left\{\tau_{m}\right\}_{m=1}^{\infty}$ be the increasing sequence of all zeros of $\sin \varphi(t)$. We choose $\alpha=\pi / 2 \omega$ and the corresponding $\varepsilon=\pi / 8$, and consider the sequence $\left\{\tau_{m}, t_{m}, s_{m}\right\}$ defined by (19) to these numbers. As in the proof of Theorem 6 , using Lemmas 2, 3, and 5, we can obtain the estimates

$$
\sin ^{2} \varphi(t) \geq \begin{cases}\left(4 \omega^{2} / \pi^{2}\right) \tilde{h}\left(t ; \tau_{m}\right)^{2} & \text { if } \tau_{m} \leq t \leq t_{m} \\ 1 / 16 & \text { if } t_{m} \leq t \leq s_{m} \\ \left(4 \varepsilon^{2} / \pi^{2}\right)\left(\tau_{m+1}-t\right)^{2} & \text { if } s_{m} \leq t \leq \tau_{m+1}\end{cases}
$$

We consider $I_{n}=\left(a_{n}, b_{n}\right)$ for a fixed $n$ and suppose that $\left.a\right) \tau_{j} \in I_{n}$ for some $j \in \mathbb{N}$. By Lemma $3 t_{j-1} \notin I_{n}$ and $s_{j} \notin I_{n}$, consequently,

$$
\begin{gathered}
\int_{a_{n}}^{b_{n}} h(t) \sin ^{2} \varphi(t) \mathrm{d} t=\int_{a_{n}}^{\max \left\{a_{n} ; s_{j-1}\right\}} \ldots+\int_{\max \left\{a_{n} ; s_{j-1}\right\}}^{\tau_{j}} \ldots \\
+\int_{\tau_{j}}^{\min \left\{b_{n} ; \tau_{j}+1 /\left(1+M_{n}\right)\right\}} \ldots+\int_{\min \left\{b_{n} ; \tau_{j}+1 /\left(1+M_{n}\right)\right\}}^{b_{n}} \ldots
\end{gathered}
$$


We apply Lemmas 5, 9 and obtain

$$
\begin{aligned}
& \int_{a_{n}}^{b_{n}} h(t) \sin ^{2} \varphi(t) \mathrm{d} t \geq \frac{4 \varepsilon^{2}}{\pi^{2}} \int_{a_{n}}^{\max \left\{a_{n} ; s_{j-1}\right\}} h \\
& \quad+\frac{4 \omega^{2}}{\pi^{2}} \int_{\max \left\{a_{n} ; s_{j-1}\right\}}^{\tau_{j}} h(t)\left(\tau_{j}-t\right)^{2} \mathrm{~d} t \\
& \quad+\frac{4 \omega^{2}}{9 \pi^{2}} \int_{\tau_{j}}^{\min \left\{b_{n} ; \tau_{j}+1 /\left(1+M_{n}\right)\right\}} h(t)\left(t-\tau_{j}\right)^{2} \mathrm{~d} t \\
& \quad+\frac{4 \omega^{2}}{9 \pi^{2}} \int_{\min \left\{b_{n} ; \tau_{j}+1 /\left(1+M_{n}\right)\right\}}^{b_{n}} h(t)\left(\frac{1}{1+M_{n}}\right)^{2} \mathrm{~d} t \\
& \quad \geq c_{8} \int_{a_{n}}^{b_{n}} h(t)\left(\min \left\{\left|t-\tau_{j}\right| ; \frac{1}{1+M_{n}}\right\}\right)^{2} \mathrm{~d} t,
\end{aligned}
$$

where

$$
c_{8}:=\min \left\{\frac{1}{16} ; \frac{4 \omega^{2}}{9 \pi^{2}}\right\} .
$$

b) If $\tau_{j} \leq a_{n}<t_{j}$, then we know that $s_{j}>b_{n}$. Using $\tilde{h}\left(t ; \tau_{j}\right) \geq \tilde{h}\left(t ; a_{n}\right)$ and repeating the computation in a) with $a_{n}$ instead of $\tau_{j}$ we get

$$
\int_{a_{n}}^{b_{n}} h(t) \sin ^{2} \varphi(t) \mathrm{d} t \geq c_{8} \int_{a_{n}}^{b_{n}} h(t)\left(\min \left\{t-a_{n} ; \frac{1}{1+M_{n}}\right\}\right)^{2} \mathrm{~d} t .
$$

c) If $\tau_{j}<t_{j} \leq a_{n}$, then we know that $\tau_{j+1} \geq b_{n}$ and

$$
\begin{aligned}
& \int_{a_{n}}^{b_{n}} h(t) \sin ^{2} \varphi(t) \mathrm{d} t \geq \frac{4 \varepsilon^{2}}{\pi^{2}} \int_{a_{n}}^{\min \left\{b_{n} ; s_{j}\right\}} h \\
& \quad+\frac{4 \omega^{2}}{\pi^{2}} \int_{\min \left\{b_{n} ; s_{j}\right\}}^{b_{n}} h(t)\left(b_{n}-t\right)^{2} \mathrm{~d} t \\
& \quad \geq c_{8} \int_{a_{n}}^{b_{n}} h(t)\left(\min \left\{b_{n}-t ; \frac{1}{1+M_{n}}\right\}\right)^{2} \mathrm{~d} t .
\end{aligned}
$$

Case B: $x$ is non-oscillatory. In this case solution $x$ is monotonous for large $t$ 's, and $x(t) \rightarrow x_{\infty}, y(t) \rightarrow y_{\infty}$ as $t \rightarrow \infty$. By (10) $y_{\infty}=0$. But $r_{\infty}>0$, therefore $x_{\infty} \neq 0$ and $\varphi(t) \rightarrow 0(\bmod \pi)$. Equation $(11)$ implies that $x(t) y(t)<0$; for the sake of definiteness we suppose that $\sin \varphi(t)<0$ for $t \in\left[t_{*}, \infty\right)$. Then there is an integer $m$ such that

$$
\begin{gathered}
\varphi(t)=m \pi+\chi(t) \quad\left(-\pi<\chi(t) \leq 0, \lim _{t \rightarrow \infty} \chi(t)=0\right), \\
\chi^{\prime}(t) \leq-\omega-h(t) \chi(t) \quad\left(t_{*} \leq t<\infty\right) .
\end{gathered}
$$

The solution of the problem

$$
u^{\prime}=-\omega-h(t) u, \quad u\left(t_{*}\right)<0
$$


admits the estimate

$$
\begin{aligned}
u(t) & =e^{-\left(H(t)-H\left(t_{*}\right)\right)} u\left(t_{*}\right)-\omega \int_{t_{*}}^{t} e^{-(H(t)-H(s))} \mathrm{d} s \\
& \leq-\omega \tilde{h}\left(t ; t_{*}\right) \quad\left(t_{*} \leq t<\infty\right) .
\end{aligned}
$$

By the basic theorem of differential inequalities (for example, [2, Theorem 4.1 in Chapter III]) we get

$$
\chi(t) \leq-\omega \tilde{h}\left(t ; t_{*}\right) \quad\left(t_{*} \leq t<\infty\right)
$$

If $n$ is so large that $a_{n} \geq t_{*}$ and $\chi(t)>-\pi / 4$ for $t \geq a_{n}$, then the application of (31) and Lemma 9 yields

$$
\begin{aligned}
& \int_{a_{n}}^{b_{n}} h(t) \sin ^{2} \varphi(t) \mathrm{d} t=\int_{a_{n}}^{b_{n}} h(t) \sin ^{2} \chi(t) \mathrm{d} t \\
& \quad \geq \frac{4 \omega^{2}}{\pi^{2}} \int_{a_{n}}^{b_{n}} h(t) \tilde{h}\left(t ; a_{n}\right)^{2} \mathrm{~d} t \\
& \quad \geq \frac{4 \omega^{2}}{\pi^{2}} \int_{a_{n}}^{b_{n}} h(t)\left(\frac{1}{M_{n}}\left(1-e^{-M_{n}\left(t-a_{n}\right)}\right)\right)^{2} \mathrm{~d} t \\
& \quad \geq \frac{4 \omega^{2}}{9 \pi^{2}}\left(\int_{a_{n}}^{\min \left\{b_{n} ; a_{n}+1 / M_{n}\right\}} h(t)\left(t-a_{n}\right)^{2} \mathrm{~d} t\right. \\
& \left.\quad+\int_{\min \left\{b_{n} ; a_{n}+1 / M_{n}\right\}}^{b_{n}} h(t) \frac{1}{M_{n}^{2}} \mathrm{~d} t\right) \\
& \quad \geq c_{8} \int_{a_{n}}^{b_{n}} h(t)\left(\min \left\{t-a_{n} ; \frac{1}{1+M_{n}}\right\}\right)^{2} \mathrm{~d} t .
\end{aligned}
$$

Summing up, for the solution $x$ we have obtained the common estimate

$$
\begin{aligned}
& \int_{a_{n}}^{b_{n}} h(t) \sin ^{2} \varphi(t) \mathrm{d} t \\
& \quad \geq c_{8} \min _{\xi \in \overline{I_{n}}} \int_{a_{n}}^{b_{n}} h(t)\left(\min \left\{|t-\xi| ; \frac{1}{1+M_{n}}\right)^{2} \mathrm{~d} t .\right.
\end{aligned}
$$

By condition (29) estimate (32) implies (30), which is a contradiction.

A sharpened form of Smith's second theorem follows from Theorem 11.

Corollary 12. Suppose that there exists a sequence $\left\{I_{n}\right\}_{n=1}^{\infty}$ of non-overlapping intervals and a number $\kappa \in(0,1)$ such that

$$
\sum_{n=1}^{\infty}\left(\min \left\{\left|I_{n}\right| ; \frac{1}{1+M_{n}}\right\}\right)^{2} \int_{H_{n}} h=\infty
$$


is satisfied with arbitrary sequence $\left\{H_{n} \subset I_{n}\right\}$, where $H_{n}$ is a finite union of intervals and for the measure of $H_{n}$ there holds $\operatorname{mes}\left(H_{n}\right) \geq \kappa\left|I_{n}\right|$.

Then the zero solution of (1) is asymptotically stable.

Proof. Without loss of the generality we can suppose that $\left|I_{n}\right| \leq 2 \zeta(n \in \mathbb{N})$ with an arbitrary constant $\zeta$. This means that if (33) holds for a sequence $\left\{I_{n}\right\}$ and $\kappa$, then it also holds for another sequence $\left\{J_{m}\right\}_{m=1}^{\infty}$ with the same $\kappa$, but $\left|J_{m}\right| \leq 2 \zeta(m \in \mathbb{N})$. In fact, to prove this assertion let us observe at first the obvious fact that for arbitrary $\left\{\alpha_{n}, \beta_{n}, \gamma_{n}\right\}\left(0<\alpha_{n}<1, \beta_{n}, \gamma_{n}>0\right)$ and $\delta>0$ the two divergences

$$
\sum_{n=1}^{\infty} \min \left\{\alpha_{n} ; \beta_{n}\right\} \gamma_{n}=\infty, \quad \sum_{n=1}^{\infty} \min \left\{\alpha_{n} ; \beta_{n} ; \delta\right\} \gamma_{n}=\infty
$$

are equivalent.

If $\left|I_{n}\right|>2 \zeta$, then we divide the interval $I_{n}$ into subintervals

$$
I_{n}=\cup_{j=1}^{l_{n}} I_{n j}, \quad \zeta \leq\left|I_{n j}\right| \leq 2 \zeta, \quad I_{n j}^{\circ} \cap I_{n m}^{\circ}=\emptyset(j \neq m)
$$

$\left(K^{\circ}\right.$ denotes the inside of interval $K$ ) and write the estimate

$$
\begin{gathered}
\left(\min \left\{\left|I_{n}\right| ; \frac{1}{1+M_{n}} ; \zeta\right\}\right)^{2} \int_{H_{n}} h=\left(\min \left\{\frac{1}{1+M_{n}} ; \zeta\right\}\right)^{2} \times \\
\times \sum_{j=1}^{l_{n}} \int_{H_{n} \cap I_{n j}} h \leq \sum_{j=1}^{l_{n}}\left(\min \left\{\frac{1}{1+M_{n j}} ;\left|I_{n j}\right|\right\}\right)^{2} \int_{H_{n j}} h
\end{gathered}
$$

where the finite union $H_{n j}$ of intervals consists of the intersections of elements of $H_{n}$ with $I_{n j}$, supplied with further subintervals of $I_{n j}$, if necessary, so that $\operatorname{mes}\left(H_{n j}\right) \geq$ $\kappa\left|I_{n j}\right|$ hold. We obtain $\left\{J_{m}\right\}$ if we exchange all $I_{n}$ of the properties $\left|I_{n}\right|>2 \zeta$ with $\left\{I_{n j}\right\}_{j=1}^{l_{n}}$.

It has remained to prove that if (33) is satisfied for some $\left\{I_{n}\right\}\left(\left|I_{n}\right| \leq \pi / 2 \omega\right)$ with some $\kappa$, then condition (29) is also satisfied for the same $\left\{I_{n}\right\}$. In fact, defining

$$
\gamma_{n}:=\frac{1-\kappa}{2} \min \left\{\left|I_{n}\right| ; \frac{1}{1+M_{n}}\right\}
$$

we can write

$$
\begin{aligned}
& \int_{a_{n}}^{b_{n}} h(t)\left(\min \left\{\left|t-\xi_{n}\right| ; \frac{1}{1+M_{n}}\right\}\right)^{2} \mathrm{~d} t \\
& \geq \gamma_{n}^{2} \int_{\min \left\{a_{n} ; \xi_{n}-\gamma_{n}\right\}}^{\xi_{n}-\gamma_{n}} h+\gamma_{n}^{2} \int_{\min \left\{\xi_{n}+\gamma_{n} ; b_{n}\right\}}^{b_{n}} h \\
& \quad=\frac{(1-\kappa)^{2}}{4}\left(\min \left\{\left|I_{n}\right| ; \frac{1}{1+M_{n}}\right\}\right)^{2} \int_{H_{n}} h
\end{aligned}
$$


and

$$
\operatorname{mes}\left(H_{n}\right) \geq\left|I_{n}\right|-2 \gamma_{n} \geq\left|I_{n}\right|-(1-\kappa)\left|I_{n}\right|=\kappa\left|I_{n}\right|,
$$

which concludes the proof.

Example 13. Consider an arbitrary sequence of non-overlapping intervals $\left\{I_{n}\right\}_{n=1}^{\infty}$ and a function $h:[0, \infty) \rightarrow[0, \infty)$ such that

$$
\left|I_{n}\right|=\frac{1}{n^{\alpha}}, \quad h(t)=n^{\beta}\left(t \in I_{n}\right) \quad(\alpha \in \mathbb{R}, \beta \in \mathbb{R} ; n \in \mathbb{N}) .
$$

We are looking for conditions on $\alpha, \beta$ guaranteeing asymptotic stability. We apply condition (33) with $\kappa=1 / 2$.

Case a) $1 / n^{\alpha} \leq 1 /\left(1+n^{\beta}\right)$ :

$$
J_{n}:=\left(\min \left\{\frac{1}{n^{\alpha}} ; \frac{1}{1+n^{\beta}}\right\}\right)^{2} \int_{H_{n}} n^{\beta} \mathrm{d} t \geq\left(\frac{1}{n^{\alpha}}\right)^{2} \frac{1}{2 n^{\alpha}} n^{\beta}=\frac{1}{2} n^{\beta-3 \alpha} .
$$

To get divergence $(33)$ we demand $-(\beta-3 \alpha) \leq 1$, that is

$$
(\alpha, \beta) \in Q_{a}:=\left\{(\alpha, \beta) \in \mathbb{R}^{2}: 0<\alpha<\frac{1}{2}, 3 \alpha-1 \leq \beta<\alpha\right\} .
$$

Case b) $1 / n^{\alpha}>1 /\left(1+n^{\beta}\right)$ and $\beta \geq 0$ :

$$
J_{n} \geq\left(\frac{1}{1+n^{\beta}}\right)^{2} \frac{1}{2 n^{\alpha}} n^{\beta} \geq\left(\frac{1}{2 n^{\beta}}\right)^{2} \frac{1}{2 n^{\alpha}} n^{\beta}=\frac{1}{8} n^{-(\beta+\alpha)} .
$$

Now we require $\alpha+\beta \leq 1$, i.e.,

$$
\begin{gathered}
(\alpha, \beta) \in Q_{b}:=Q_{b 1} \cup Q_{b 2}, \\
Q_{b 1}:=\left\{(\alpha, \beta) \in \mathbb{R}^{2}: \alpha \geq 0, \alpha \leq \beta \leq-\alpha+1\right\}, \\
Q_{b 2}:=\left\{(\alpha, \beta) \in \mathbb{R}^{2}: \alpha \leq 0,0 \leq \beta \leq-\alpha+1\right\} .
\end{gathered}
$$

Case c) $1 / n^{\alpha}>1 /\left(1+n^{\beta}\right)$ and $\beta<0$ :

$$
J_{n} \geq\left(\frac{1}{1+n^{\beta}}\right)^{2} \frac{1}{2 n^{\alpha}} n^{\beta} \geq \frac{1}{2^{2}} \frac{1}{2 n^{\alpha}} n^{\beta}=\frac{1}{8} n^{\beta-\alpha} .
$$

In order to obtain $(33)$ we assume $-(\beta-\alpha) \leq 1$, i.e.,

$$
(\alpha, \beta) \in Q_{c}:=\left\{(\alpha, \beta) \in \mathbb{R}^{2}: \alpha \leq 0, \alpha-1 \leq \beta<0\right\} .
$$

If $Q:=Q_{a} \cup Q_{b} \cup Q_{c}$, then

$$
\begin{aligned}
Q= & \left\{(\alpha, \beta) \in \mathbb{R}^{2}: \alpha \leq 0, \alpha-1 \leq \beta \leq-\alpha+1\right\} \\
& \cup\left\{(\alpha, \beta) \in \mathbb{R}^{2}: 0 \leq \alpha \leq \frac{1}{2}, 3 \alpha-1 \leq \beta \leq-\alpha+1\right\} .
\end{aligned}
$$

By Corollary 12 we have proved the following assertion: Let $h$ and $\left\{I_{n}\right\}$ be defined by (34). If $(\alpha, \beta) \in Q$ (see Figure 1), then the zero solution is asymptotically stable. 


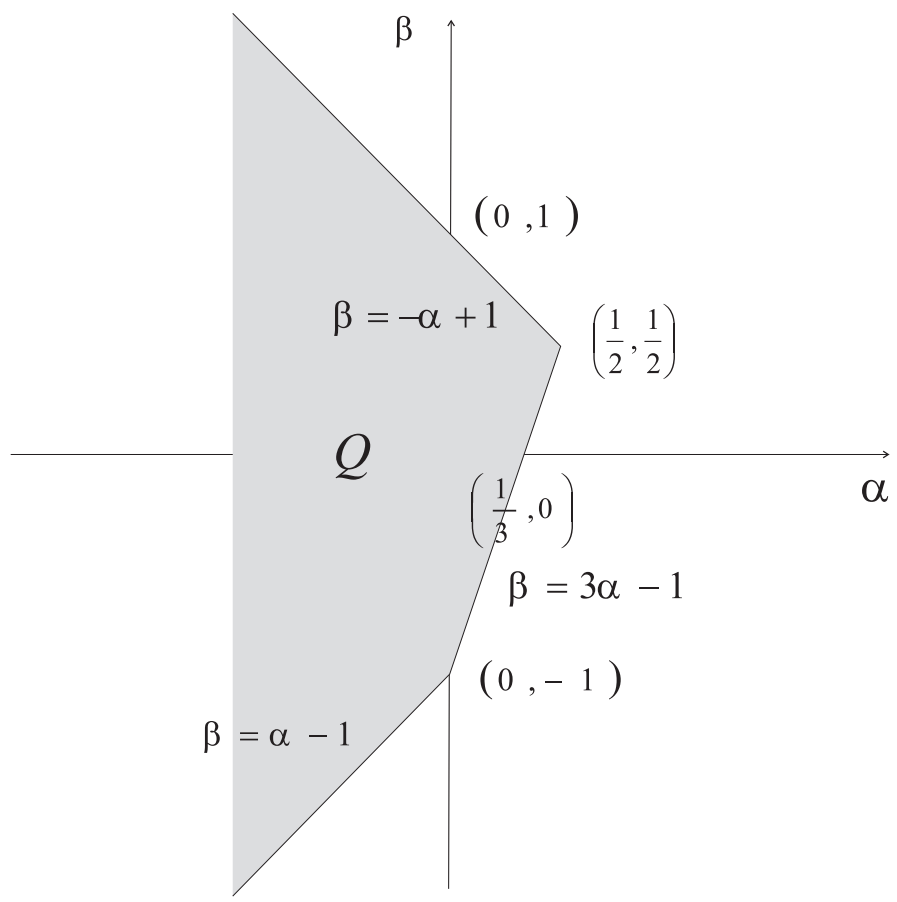

Figure 1: The domain of asymptotic stability in Example 13

Remark 14. P. Pucci and J. Serrin [13] have established a theory for global asymptotic stability for very general nonlinear second order systems of many degrees of freedom. Their study was based on a variational inequality and Lyapunov's second method. Later on [14] they apply their theory to the problem of intermittent damping. The specializations of their main results to the damped linear oscillator (see Corollaries 3 and 4) and our Theorem 11 and Corollary 12 are independent. Beyond the generality, the advantage of their corollaries in comparison with our results is that they can use the integral mean of the coefficient $h$ instead of its supremum on interval $I_{n}$. However, we do not demand the positivity of the infimum of the coefficient. Corollaries 3 and 4 in [14] can be applied to Example 13 only in the case $\beta=0$. If we modify definition (34) so that $h(t)=0$ for rational $t$ 's, then neither Pucci's and Serrin's results nor Smith's Theorem B can be applied to Example 13, although the assertion and its proof remains valid by Corollary 12 .

Remark 15. The condition $\kappa<1$ in Corollary 12 is sharp. In fact, if $h$ is bounded on $[0, \infty)$ and $\int_{0}^{\infty} h=\infty$, then condition (33) is satisfied with $\kappa=1$ and $I_{n}=[n-1, n)$. If Corollary 12 were true with $\kappa=1$, then the zero solution would be asymptotically stable, but this assertion is false $[9,11,14]$. 
Remark 16. If $I_{n}=\left(a_{n}, b_{n}\right)$, and one wants to rid Theorem 11 of the condition $\left|I_{n}\right|=b_{n}-a_{n} \leq \pi / 2 \omega(n \in \mathbb{N})$, then one has to involve $a_{n}=\xi_{n, 0}<\xi_{n, 1}<\ldots<$ $\xi_{n, p_{n}}<\xi_{n, p_{n}+1}=b_{n}$ into condition (17) instead of $\xi_{n}$ in the form $\left|t-\xi_{n, j}\right|$ so that

$$
\xi_{n, j+1}-\xi_{n, j} \geq \alpha \quad\left(n=1,2, \ldots ; j=1, \ldots, p_{n-1}\right)
$$

with some constant $\alpha \in[\pi / 2 \omega, \pi / \omega)$. The proof follows the line of the proof of Theorem 11; we leave the details to the reader.

\section{ACKNOWLEDGMENTS}

Supported by the Hungarian National Foundation for Scientific Research (OTKA K109782).

\section{REFERENCES}

[1] Z. Artstein, E. F. Infante, On the asymptotic stability of oscillators with unbounded damping, Quart. Appl. Math., 34 (1976/77), 195-199.

[2] P. Hartman, Ordinary Differential Equations, Birkhäuser, Boston, Mass., 1982.

[3] L. Hatvani, A generalization of the Barbashin-Krasovskij theorems to the partial stability in nonautonomous systems, Qualitative Theory of Differential Equations, Vol. I, II (Szeged, 1979), 381-409, Colloq. Math. Soc. János Bolyai, 30, North-Holland, Amsterdam-New York, 1981.

[4] L. Hatvani, Nonlinear oscillation with large damping, Dynam. Systems Appl., 1 (1992), 257-269.

[5] L. Hatvani, Integral conditions on the asymptotic stability for the damped linear oscillator with small damping, Proc. Amer. Math. Soc. 124 (1996), 415-422.

[6] L. Hatvani, On the damped harmonic oscillator with time dependent damping coefficient, J. Dynam. Differential Equations, 30 (2018), DOI: 10.1007/s10884017-9597-z.

[7] L. Hatvani, T. Krisztin, Necessary and sufficient conditions for intermittent stabilization of linear oscillators by large damping, Differential Integral Equations, 10 (1997), 265-272.

[8] L. Hatvani, T.Krisztin, V. Totik, A necessary and sufficient condition for the asymptotic stability of the damped oscillator, J. Differential Equations 119 (1995), 209-223. 
[9] L. Hatvani, V. Totik, Asymptotic stability of the equilibrium of the damped oscillator, Differential Integral Equations, 6 (1993), 835-848.

[10] J. Karsai, On the asymptotic behaviour of the solutions of a second order linear differential equation with small damping, Acta Math. Hungar., 61 (1993), 121127.

[11] J. Karsai, On the asymptotic behaviour of solutions of certain nonlinear differential equations, Proceedings of the Eleventh International Conference on Nonlinear Oscillations, (Budapest, 1987), 416-419, János Bolyai Math. Soc., Budapest, 1987.

[12] J. J. Levin, J. A. Nohel, Global asymptotic stability for nonlinear systems of differential equations and applications to reactor dynamics, Arch. Ration. Mech. Anal., 5 (1960), 194-211.

[13] P. Pucci, J. Serrin, Precise damping conditons for global asymptotic stability for nonlinear second order systems, Acta Math., 170 (1993), 275-307.

[14] P. Pucci, J. Serrin, Asymptotic stability for intermittently controlled nonlinear oscillators, SIAM J. Math. Anal., 25 (1994), 815-835.

[15] P. Pucci, J. Serrin, Precise damping conditons for global asymptotic stability for nonlinear second order systems. II, J. Differential Equations, 113 (1994), $505-534$.

[16] R. A. Smith, Asymptotic stability of $x^{\prime \prime}+a(t) x^{\prime}+x=0$, Quart. J. Math. Oxford Ser. (2), 12 (1961), 123-126.

[17] J. Sugie, Smith-type criterion for the asymptotic stability of a pendulum with time-dependent damping, Proc. Amer. Math. Soc., 141 (2013), 2419-2427.

[18] J. Sugie, M. Onitsuka, Growth conditions for uniform asymptotic stability of damped oscillators, Nonlinear Anal., 98 (2014), 83-103.

[19] J. Sugie, Asymptotic stability of coupled oscillators with time-dependent damping, Qual. Theory Dyn. Syst. (2015), doi:10.1007/s12346-015-0175-7.

[20] W. Zheng, J. Sugie, Parameter diagram for global asymptotic stability of damped half-linear oscillators, Monatsh. Math., 179 (2016), 149-160. 